\title{
Richard Marienstras, La Cité et la Forêt
}

\section{Richard Marienstras}

\section{(2) OpenEdition Journals}

Édition électronique

URL : http://journals.openedition.org/shakespeare/1648

DOI : $10.4000 /$ shakespeare. 1648

ISSN : 2271-6424

Éditeur

Société Française Shakespeare

Édition imprimée

Date de publication : 1 mars 2011

Pagination : 257-272

ISBN : 2-9521475-7-4

Référence électronique

Richard Marienstras, «Richard Marienstras, La Cité et la Forêt 》, Actes des congrès de la Société

française Shakespeare [En ligne], 28 | 2011, mis en ligne le 15 février 2011, consulté le 20 avril 2019. URL : http://journals.openedition.org/shakespeare/1648; DOI : 10.4000/shakespeare.1648 


\section{H OMMAGE À RICHARd MARIENSTRAS}

Lecture de Shakespeare au XXI' siècle ${ }^{1}$

$\mathrm{N}$

ous avons un intervenant dont nous allons nous faire les interprètes puisqu'il a choisi aujourd'hui de nous écouter. Le thème de ce colloque «Shakespeare et la Cité » lui revient, il remonte à l'époque où nous conduisions ensemble le séminaire du CERLA, un des sujets que nous avions abordés entre « La Guerre » et «L'Amitié ». Après avoir été mon directeur de recherche, Richard Marienstras m'avait conviée à cette co-direction sur un pied d'égalité entre nous, et entre nos deux établissements, par un double sceau Amiens-Charles v. Mais mon premier souvenir de lui n'est pas tout à fait académique : certains d'entre vous ont eu l'occasion de revoir récemment deux interviews d'Orson Welles et Peter Brook qu'il avait réalisées pour la télévision. Oui Richard est d'abord un homme de théâtre : pour moi, son meilleur rôle, c'était un Midsummer Night's Dream jazzé très iconoclaste qu'il avait mis en scène au Centre Culturel Américain avec les étudiants que nous étions, où il avait fini par assurer le rôle de Thisbé, à la grande stupeur de ses collègues dans l'auditoire. Thisbé n'avait pas de barbe, mais une voix qui muait en cours de réplique. Ce fut un triomphe.

Mais revenons à la Cité. Vous connaissez les travaux de Richard, son engagement politique au meilleur sens du terme dans la pensée, son dialogue érudit et sensible avec le texte et l'univers shakespearien.

Je lui donne la parole :

«On voit d'emblée ce qui sépare les spectacles tragiques (ou prétendus tels) mis en scène aujourd'hui et ceux qui étaient représentés à Athènes, ceux qui furent joués à l'époque élisabéthaine, et à l'époque classique française ainsi qu'au XIX ${ }^{\text {e }}$ siècle (dans les créations d'auteurs tels qu'Ibsen ou Büchner).

D'abord, la présence inéluctable de la cité et de son rôle. Les représentations théâtrales modernes sont d'emblée mondialisées. Bien qu'écrites pour un public particulier, elles franchissent aisément les

\footnotetext{
${ }^{1}$ Texte lu par Dominique Goy-Blanquet.
} 
frontières et ne peuvent par conséquent évoquer que des problèmes et des questions que leur universalité déracine et qui flottent dans un no man's land où les sens qui s'accrochent à elles ou qu'elles se donnent au passage et en passant sont privés de stabilité, de sorte qu'elles ne peuvent plus provoquer durablement ou profondément la terreur et la pitié.

L'autre obstacle que rencontrent les productions contemporaines qui se veulent tragiques est le caractère indicible de l'horreur qu'ont subie et vécue en un siècle des millions d'hommes sur toute la planète et que le théâtre ne peut ni reproduire, ni imiter, ni même évoquer de façon véridique ou convaincante. Aucune fiction n'a le pouvoir de donner l'illusion qu'elle égale ou dépasse imaginativement l'intense inhumanité des événements dont presque tous les spectateurs gardent le souvenir et qui continuent d'ailleurs à se reproduire régulièrement dans la réalité sous des formes voisines ${ }^{2}$. [...]

[L]a configuration du monde moderne crée de grands obstacles à la possibilité même de la tragédie, si elle ne rend pas la tragédie quasiment impossible. Créer un monde fictif dont le spectacle l'emporterait en vérité — c'est-à-dire en horreur — sur les événements dont notre siècle a été à la fois le témoin et la victime : la chose ne paraît même pas imaginable.

Telle est la situation où nous nous trouvon $\mathrm{s}^{3} \gg$ conclut Richard. Dans un «au-delà de la tragédie. Pour qu'elle revienne au monde, il faudra que le monde se civilise à nouveau ${ }^{4}$. »

\section{Lecture de la « Notice » de Coriolan ${ }^{5}$}

«Dans Coriolan, malgré leurs violents conflits, les patriciens et les plébéiens partagent une même vision du corps politique. C'est pourquoi, dans la première scène de la pièce, la fable des membres et de l'estomac rapportée par Ménénius, n'est pas seulement une tirade idéologique visant à faire accepter à la plèbe le gouvernement injuste des patriciens qui les affament, bien qu'elle soit cela aussi. Cette tirade,

\footnotetext{
${ }^{2}$ Shakespeare au XXI siècle: Petite introduction aux tragédies, Éditions de Minuit, 2000, pp. 13-14.

3 Ibid., p. 15 .

${ }^{4}$ Ibid., p. 24.

5 Texte lu par Frédéric Picco.
} 
qui livre l'une des interprétations essentielles de la tragédie, a une vertu incantatoire dont la rhétorique vise à faire sentir le rôle crucial joué par le mythe dans la constitution du sentiment national. Les citoyens sont tous liés par une émotion naturelle. Leurs inimitiés passagères n'iront jamais jusqu'à remettre radicalement en cause le grand pacte social qui les lie entre eux et à la chose publique. La décision de Coriolan de ne pas brûler Rome, de l'épargner au risque de sa propre vie, illustre rétrospectivement la force de l'incorporation évoquée au premier acte et l'inscription symbolique de la cité dans la Nature. Si Coriolan renonce à sa vengeance et consent à épargner la Ville, ce n'est pas parce qu'il est le fils obéissant —ou trop obéissant de sa mère. C'est parce qu'il cède, comme il le dit, à la voix de la nature:

$\mathrm{O}$ mère, mère !

Qu'avez-vous fait? Regardez, les cieux s'ouvrent.

Les dieux abaissent leur regard, et rient

De cette scène contre nature. Oh ! ma mère, ma mère. Oh

Vous avez pour Rome remporté une heureuse victoire,

Mais pour votre fils, croyez-moi, oh! croyez-moi,

Votre triomphe sur lui est dangereux [...]

La scène est bien contre nature : un fils debout devant sa mère agenouillée, un Romain faisant la guerre à Rome, un conquérant détourné de son but par sa famille — et une mère poussant, de façon semi-consciente, son fils à la mort. Il n'y a pas, me semble-t-il, à chercher ailleurs que dans la soumission, consciente ou inconsciente, aux liens de sociabilité et aux relations entre citoyens, le ressort dernier du comportement de la plupart des personnages. Comme le remarque la grande helléniste Nicole Loraux : «Dans cette vaste mutualité qu'est la cité romaine, la primauté revient à la relation de réciprocité qui fonde la vie communautaire. Du coup, on caractérisera sans hésiter comme profondément romain l'apologue de Ménénius, qu'il soit raconté par Tite-Live ou par Plutarque, parce que, avec un bel accord, les deux textes mettent l'accent sur la dépendance réciproque des citoyens entre eux : dans cet organisme vivant qu'est Rome, le ventre ne saurait viser à une existence autonome, mais les autres membres ne gagnent rien à s'unir tous contre lui. Pour la cité romaine, il n'est d'autre principe que la recherche de la concordia, cette concorde dont le nom même énonce la nécessité impérative pour tous de vivre ensemble, afin d'éviter que la guerre civile, affrontant les citoyen s entre 
eux, ne démembre cette totalité qu'est la civitas.» Ainsi, Coriolan jettera aux chiens sa loyauté nouvellement manifestée pour les Volsques, alors qu'Aufidius n'hésitera pas à trahir la dilection que depuis si longtemps il éprouvait pour Coriolan sans la manifester.

Pourquoi thématiser si fortement l'opposition entre les deux chefs ennemis? D'abord, pour souligner l'immense supériorité guerrière de Coriolan. Mais aussi pour débattre, une fois encore, la question de l'honneur, que Shakespeare avait déjà abordée. Et pourquoi cet intérêt pour l'honneur? Parce que c'est un attribut qui articule l'individu et la communauté politique à laquelle il appartient. Shakespeare examine sous cet angle le monde féodal dans les deux parties de Henry IV, l'Antiquité grecque dans Trö̈lus et Cressida, les origines de Rome dans Coriolan. Il s'y intéresse aussi parce que l'honneur avait jadis passé pour une valeur universelle; or, dans sa mise en question de tout ce qui a du prix, Shakespeare le disqualifie quelque peu. Dans la première partie de Henry IV, il fait dire à Falstaff : "Qu'est-ce que l'honneur? Un mot. Qu'y a-t-il dans ce mot "honneur"? Qu'est-il cet honneur? Du vent.» Dans Trö̈lus et Cressida, on voit Achille faire mourir odieusement Hector et s'en attribuer la gloire, l'honneur étant oublié. Et dans Coriolan, Aufidius, trop souvent vaincu, décide de l'emporter sur Coriolan par n'importe quel moyen. Il le fera donc assassiner à la fin de la tragédie. Ainsi, Coriolan, dont les décisions vacillent parfois, reste l'incomparable modèle des vertus romaines.

Ces vertus sont toutes sublimées par le surnom que Caius Martius, sur l'ordre de Cominius, reçoit pour rendre éternel son exploit à Corioles. Car «le premier des noms que portaient les Romains, comme Caius, était leur propre; le second, comme Martius, était le nom de la famille et maison; et le troisième était un surnom, qui se donnait ou pour quelque acte, ou quelque aventure notable, ou pour quelque marque de la face et forme du corps, ou pour quelque vertu ». Le surnom de Coriolan fut proclamé par un héraut à toute la cité après sa victoire.

Il y a un autre aspect de l'honneur, lié à la scène du Forum où Coriolan hésite à quémander les voix du peuple (II.iii) en jouant sur les divers sens du mot voix (le son émis, le truchement, les mots ou le langage, le jugement, le vote) : Coriolan refuse alors de se prêter à la coutume qui consiste à montrer les blessures reçues dans les combats 
pour Rome. On aura remarqué la force et, conjointement, la faiblesse de la vox populi dans la pièce. Capable de faire exiler Coriolan, elle s'amenuise lamentablement lorsqu'il revient à Rome à la tête de l'armée volsque. Shakespeare, d'ailleurs, fait exprimer à Coriolan le mépris que le peuple inspire au héros : rien de ce que le peuple peut lui attribuer ne saurait le satisfaire. L'honneur décerné par la voix populaire est inacceptable. Il est également clair que l'haleine fétide que Coriolan attribue au peuple ne saurait être une source d'honneur.

Chez Sénèque, chez Épicure, chez Montaigne, la vanité de ce qui donne de l'honneur au-delà de l'action honorable elle-même est fortement soulignée. L'honneur, en effet, est décerné ou porté par la réputation ou la renommée, mais les voix qui font la renommée doivent être celles d'hommes vertueux. L'éloge prononcé par n'importe qui n'est qu'un son lancé en l'air et ne mérite pas d'être retenu comme un bien. Et puisque la gloire et l'honneur viennent de la renommée, et que la renommée vient de l'opinion, la renommée d'un homme est ce que le peuple dit de lui : d'emblée, la renommée est ainsi liée à l'opinion et à la rumeur, il faut donc la fuir plutôt que la rechercher ${ }^{6}$. »

\section{Lecture de Le proche et le lointain ${ }^{7}$}

«L'animal et le hors la loi »: «C'est ainsi que Manwood rappelle deux pratiques juridiques qui confirment le caractère sacré de la forêt et sa spécificité physique et légale. Il importe d'abord, dit-il, que les limites de la forêt soient connues de tous. En effet, si un homme vient à être accusé d'avoir tué un animal de la forêt, le lieu où l'animal a été abattu est essentiel à la cause : «S'il est tué hors de la Forêt [...], cela peut ne pas être une infraction aux lois de la Forêt » (VII, 2, p.129).

De même, si un forestier aperçoit un homme dans la forêt «et, après qu'à cor et à cri on lui a ordonné de se rendre, qu'il ne se rend pas mais s'enfuit ou résiste », alors, si le forestier tue le contrevenant dans les limites de la forêt, il ne sera pas accusé de meurtre. Car tuer un contrevenant «dans les limites de la forêt ou hors de ces limites fait, ou ne fait pas, de l'homicide un crime» (VII, 2, p. 130). Dans la forêt, le contrevenant rétif se place dans la situation de l'animal sauvage chassé

\footnotetext{
${ }^{6}$ Notice de Coriolan, Shakespeare: Tragédies, éd. J-M. Déprats, Paris, Gallimard, coll. «La Pléiade», 2002, pp. 1559-1562.

7 Texte lu par Catherine Lisak.
} 
par le roi : sa mise à mort est légitime. L'expression utilisée est after hue and cry made to him, "après qu'à cor et à cri on a manifesté qu'on le poursuivait », ou, comme dit Littré, qu'on allait, « au son du cor et à la huée, comme pour les bêtes fauves», se lancer à sa poursuite. «Le délit doit être commis dans la Forêt», confirme Edward Coke pour qu'on puisse faire retentir ces verba dolentis. L'expression renvoie à une pratique très ancienne de poursuite des criminels : l'appel à la poursuite pouvait être lancé par des particuliers, des constables ou des officiers de paix. L'officier de paix agissait alors comme s'il avait détenu le mandat d'un magistrat: son action était donc faite au nom du roi. Ceux qui se joignaient à lui avaient le droit d'appréhender l'homme poursuivi -, qui pouvait éventuellement plus tard faire reconnaître son innocence. S'il se réfugiait dans une maison, on pouvait en forcer la porte au cas où l'entrée en était refusée. L'homme chassé « à cor et à cri » se trouvait ainsi dans un espace sauvage où le droit commun du royaume n'était pas applicable. L'homme devenait hors-la-loi, chasseur, devenu gibier, homme «portant une tête de loup », selon la définition de Bracton rappelée par Cowell, et qui a perdu le bénéfice de la protection du roi et des lois. Tous les habitants du comté devaient aider l'officier de paix dans sa poursuite, faute de quoi ils étaient collectivement mis à l'amende par la cour locale. Toute la meute des hommes à ses trousses, le délinquant est «ensauvagé » sur l'entier territoire de la forêt, de telle sorte, dit Manwood, que «de bourg en bourg, de village en village et d'un endroit à l'autre à l'intérieur des limites et des bornes de la Forêt » (XIX, 6, p. 313) le braconnier soit poursuivi sans relâche.

L'homme poursuivi ne trouvera un refuge provisoire qu'à l'extérieur de la forêt: là, soumis à nouveau au droit commun du royaume, il ne pourra être appréhendé que sur mandat. Cependant, et c'est là une clause capitale, de même que les chiens, s’ils tenaient le gibier et étaient entraînés par lui, pouvaient être suivis par l'habitant du purlieu à l'intérieur de la forêt pour achever la poursuite, de même le forestier, s'il ne perd pas de vue le contrevenant, pourra le suivre à l'extérieur de la forêt pour l'appréhender.

Ces deux poursuites, symétriques et inverses, permettent de déterminer des lieux de la sauvagerie, qui ne sont pas seulement situés de manière topographique, mais qui résultent d'une configuration complexe où entrent : 1) une relation spatiale ;2) une relation à la loi ; 
3) une relation à la sauvagerie et au sacré ; 4) une relation entre deux agents dont la polarité est tantôt celle du chasseur et du gibier, tantôt celle de la meute et du gibier, tantôt celle du propriétaire à l'animal possédé - et qui peut aussi disparaître quand change l'enceinte où la poursuite se déroule. La polarité est bien mise en relief par Manwood qui précise qu'en cas d'infraction aux lois de la forêt il ne peut y avoir d'agent principal et de complices: tous sont considérés comme les agents principaux de la transgression si elle est commise à plusieurs : None are accessories in the trespasses of the Forest, but all are principals (XIX, v, p. 312) : «Dans les délits forestiers, nul n'est agent accessoire, chacun est agent principal. ${ }^{8}$

«Othello a absorbé un poison qui lui fait croire qu'il est changé en bête (IV.i.62), mais aussi qui le transforme en un être sauvage et écumant, comme l'explique Iago au cours de la crise d'épilepsie :

Cette léthargie doit suivre tranquillement son cours,

Sinon, il écume, et bientôt une sauvage démence

S'empare de lui.

(IV.i.53-55)

Desdémone ne le reconnaît plus. Quant à elle, elle est devenue à ses yeux une putain, une femme qui ne sait pas distance garder. Rapprochée de tous, elle est au contraire éloignée de lui. Des écarts nouveaux sont établis. Shakespeare a fait en sorte que les signes visibles et ce qu'ils manifestent se sont séparés, que l'être et l'apparence, les choses et leur valeur ne correspondent plus. C'est le principe même de Iago :

Il me faut déployer le pavillon et les signes de l'affection,

Mais ce ne sont vraiment que signes.

(I.i.157-57)

Dans la scène où Othello traite Desdémone en prostituée et Émilie en entremetteuse, les choses sont agencées de telle sorte que ce qui se passe est compris différemment par chaque personnage et par les spectateurs. L'épouse fidèle se voit prise dans un rôle qu'elle ne comprend pas, si bien que la putain imaginée par Othello et la vraie Desdémone dont il n'entend pas les dénégations finissent par se superposer dans l'esprit du spectateur. La vérité et sa déformation qui est la vérité d'Othello - se constituent en une réalité unique, en une

${ }^{8}$ Le proche et le lointain, Éditions de Minuit, 1981, ch. I, 6. «L'animal et le hors la loi », pp. 46-48. 
image dramatique parfaitement unifiée. Ainsi, les êtres et les choses ne se comprennent peut-être que dans le cadre de la vision qui leur donne un sens : et ce sens, alors, n'est pas comme ajouté à la réalité : il s'incorpore à elle ${ }^{9}$. »

«Le suicide d'Othello est moins destiné à lui permettre de «se donner une contenance» ou à montrer que Iago a su entièrement s'emparer de son âme qu'à lui permettre de se faire justice d'une main vénitienne, en tuant en lui le « chien circoncis» :

Et dites en outre qu'un jour, dans Alep,

Alors qu'enturbanné et malfaisant, un Turc,

Battait un Vénitien et insultait Venise,

J'ai saisi par la gorge ce chien circoncis

Et je l'ai frappé ainsi.

$$
\text { (Il se poignarde) }
$$

Car il est un homme

$$
\text { dont la main }
$$

Comme celle du vil Indien, jeta une perle

Plus précieuse que toute sa tribu...

(V.ii.347-49)

Dans les deux Quartos de l'époque, c'est le terme Indian qui apparaît. Mais dans le Folio, on lit Iudean, «Judéen » ou «Juif ». Les spécialistes ont interminablement débattu pour savoir laquelle des deux versions était la bonne. L'hésitation est elle-même significative : ce qu'Othello tue à la fin de la pièce pour avoir rejeté la perle unique, la source de vie, c'est à la fois l'Indien, le Juif et le Turc, c'est-à-dire des êtres que l'idéologie dominante situait hors de la loi naturelle et dont la proximité risquait de subvertir les valeurs de la Cité. Mais Shakespeare situe à l'intérieur de celle-ci l'essentiel de la menace. Opaque, indéchiffrable, condamné à la torture lente, Iago le traître est un emblème de l'ennemi principal qui utilise la menace extérieure pour parvenir à ses fins. Le but profond de sa machination n'est pas de détruire un mariage : c'est d'empêcher l'accomplissement d'un amour exceptionnel et exemplaire dont les normes dépassent les exigences de la coutume et défient les haines accumulées contre les étrangers. Ce qui est ainsi suggéré, c'est que l'appartenance à l'humanité ne saurait se

\footnotetext{
${ }^{9}$ Ibid., ch. VI, 14, « La métamorphose d'Othello », p. 232.
} 
définir par l'appartenance territoriale, nationale ou religieuse : elle se définit par le refus d'un refus.

Nul ne peut dire quels sont les hommes qui ont le droit de vivre et d'aimer sur la terre et quels sont les hommes privés de ce droit. Le crime de Iago, c'est d'abord le refus général qu'il a soulevé en lançant, contre Othello, la rumeur de Venise, se dressant, par là même, contre tous les hommes.

Shakespeare s'est servi du rapprochement des civilisations pour montrer que le pire ennemi n'est pas toujours le plus apparent. L'homme n'a pas à chercher au loin ce qui le détruit : il est assuré de le trouver sur place, et l'amour, sur quoi les communions se fondent, est le lieu privilégié du crime $\mathrm{e}^{10}$.»

\section{Lecture de Shakespeare au XXI'e siècle}

«Les écarts entre les hommes sont aussi de l'ordre du politique. Il n'y a guère de politique shakespearienne, mais une manière de leçon ou d'avertissement : gouverner est difficile, sinon impossible. Car le Prince n'a que rarement à choisir entre une bonne et une mauvaise décision, mais plutôt entre des décisions également ou inégalement mauvaises. Shakespeare met en scène la fin de l'angélisme politique. En termes d'époque, cela signifiait que ni la Providence, ni les astres, ni la vertu, ni la sagesse, ni la connaissance, ni le cynisme (ou le machiavélisme «noir») ne conduisent infailliblement l'action vers le succès. C'est de la Fortune qu'il faut se rendre maître, et la Fortune - comme on le disait depuis des siècles — est inconstante et volage. Ainsi la décrit Fluellen :

La Fortune est représentée aveugle, un bandeau sur les yeux, pour vous signifier que la Fortune est aveugle; en outre, elle est représentée avec une roue pour vous signifier, et c'est la morale de la chose, qu'elle est changeante, et inconstante, qu'elle n'est que mutabilité, et variation; et son pied, voyez-vous, est fixé sur une pierre sphérique, qui roule, et roule et roule. (Henri $V$, III.vi.30-36)

De plus, le passage à l'acte n'a pas d'assises rationnelles, c'est un saut, un élan vers l'inconnu. Hamlet, malgré quelques interprétations romantiques attardées, n'est pas — entre autres choses — une pièce où

${ }^{10}$ Ibid., pp. 236-7.

${ }^{11}$ Texte lu par Dominique Goy-Blanquet. 
hésite un homme incapable d'agir, c'est un débat sur l'impossibilité d'asseoir rationnellement l'action. Ce qui ne signifie pas que pour agir, on puisse s'en remettre aveuglément au hasard, à la Fortune, à la Providence ou à l'humeur...

Tout cela peut sembler banal aujourd'hui : ça ne l'était pas à l'époque élisabéthaine. Shakespeare n'est pas un doctrinaire, mais un auteur dramatique et un poète : l'originalité de sa dramaturgie tient à ce qu'il a inventé nombre de situations concrètes où l'écheveau de ce qui pèse sur la décision nous est donné à voir sans être démêlé ni hiérarchisé. La difficulté d'agir et de gouverner vient de la nature de l'homme agissant et de la nature du monde en mouvement qu'il transforme par son action et qui le transforme. Pour la première fois, le personnage de théâtre et la société théâtrale se modifient l'un l'autre à mesure que se déroule l'action : le monde et l'individu ne sont faits que de variables, et ce qui, dans le monde ou chez l'homme, est désigné comme immuable, est moins un attribut de l'homme ou du monde qu'une vision - une interprétation — de certains agents de l'action. Les seules certitudes sont les plus élémentaires: le sexe, l'argent, la passion, la souffrance et la mort.

Si le stable et l'immuable font partie des illusions que les protagonistes se plaisent à entretenir, les modifications qu'ils subissent touchent au cœur même de l'action dramatique. Clairement défini dans l'exposition, ou la première phase de la tragédie, le héros subit quelque métamorphose pendant la seconde: il tend à devenir sa propre antithèse. Que ce soit Lear ou Macbeth, Othello ou Brutus, Hamlet ou Coriolan, Timon ou Titus Andronicus, l'opacité ou la duplicité originelle $\mathrm{du}$ protagoniste rendent sa transformation à la fois vraisemblable et surprenante —infiniment pitoyable ou déplorable.

La possibilité même de cette métamorphose ouvre les portes d'un théâtre de la violence - violence physique, comme dans Titus Andronicus, Le roi Lear, Othello ou Macbeth, violence psychique ou morale dans toutes les tragédies. Dans La duchesse de Malfi de Webster, la transformation de Ferdinand en loup-garou est comme l'emblème du surgissement de la violence, le chaos social et individuel formant le terreau de la tragédie. À son propos, mais pas à son propos seulement, on peut redire avec Gerhard Nebel et Paul Ricœur : 
« l'égarement fatal du héros plonge dans un mystère d'iniquité qui est le tragique même de l'être ${ }^{12} »$.

\section{Lecture de Shakespeare, la Renaissance et l'amitié13}

Shakespeare, la Renaissance et l'amitié ${ }^{14}$ est en fait l'aboutissement d'un séminaire et d'un colloque qui, comme Richard Marienstras le dit à la fin de l'Introduction, «ne furent pas seulement une étude sur l'amitié, mais une célébration de l'amitié » (p. 20).

Avant de donner quelques pages tirées de la fin de l'article, une rapide mise en perspective s'impose, au moyen de citations et de paraphrases tirées de l'Introduction, également due à Richard Marienstras ${ }^{15}$.

«La Renaissance est au confluent de discours et de traditions multiples» (p. 13) [sur l'amitié, tant antiques que médiévales]. La tradition la plus importante pour ce qui suit, et en relation avec le thème de notre Congrès, est la conception d'Aristote, selon laquelle la philia, l'amitié parmi les citoyens, est fondamentalement nécessaire au bien-être de la Cité. Pour les Grecs, nous dit Hannah Arendt dans Men in Dark Times (1968), « l'essence de l'amitié résidait dans le discours, car seul l'échange constant de paroles pouvait unir les citoyens dans la polis. Par le discours, l'importance politique de l'amitié et de l'humanité qui lui était propre se trouvaient manifestées » (p. 9).

Après un Moyen Âge qui manifeste une certaine «méfiance visà-vis de l'amitié purement humaine» (p. 12), «la Renaissance voit refleurir les textes faisant l'éloge de l'amitié [... mais] elle en produit d'autres qui désespèrent que l'homme puisse parvenir à rencontrer un ami digne des descriptions antiques, ou qui doutent que de telles amitiés soient possibles». On pense naturellement aux nombreux textes relatifs à la difficulté de distinguer l'ami véritable du flatteur, mais «d'autres textes [tels que les polémiques sur l'usure] venaient également saper la confiance que l'on pouvait avoir dans la fraternité humaine» (p. 15). «Alors que l'usure était interdite et réprouvée

\footnotetext{
12 Shakespeare au XXI siècle, pp. 69-72.

13 Texte lu par Michèle Vignaux.

${ }^{14}$ Richard Marienstras et Dominique Goy-Blanquet (éd.) : Shakespeare, la Renaissance et l'amitié, Presses de l'UFR de langues de l'Université de Picardie, collection Sterne, 1998

15 «Amitié et Démesure dans Timon d’Athènes », in ibid., pp. 277-83.
} 
pendant tout le Moyen Âge, la Réforme amorce un mouvement qui tend à autoriser le prêt à intérêt à condition que l'intérêt soit limité et "raisonnable". [...] Car si toute la richesse est convertie en dons, il ne peut y avoir de marché ni d'échange avec l'extérieur. Mais si toute la richesse devient marchandise, il ne peut plus y avoir de dons, et la communauté elle-même risque de périr » (p. 20).

Dans Timon d'Athènes, le discours de l'amitié est étroitement lié à ceux de la mesure et du don - question sur laquelle achoppent de nombreux discours sur l'amitié (p. 14). La pièce offre d'abord «le spectacle d'un [Timon] qui comble son entourage de bienfaits, qui manifeste une munificence et une générosité peu communes, et chez qui il y a beaucoup à admirer» (p. 273) — jusqu'à l'intervention de l'intendant, dont le monologue (I.ii.189-98) révèle la fragilité de la situation financière de Timon (p. 275), ce qui conduit le spectateur à adopter un autre point de vue sur la générosité de Timon, à se demander si elle ne serait pas une façon d'affirmer sa supériorité sur autrui dans la mesure où elle n'admet pas de réciprocité (p. 276), et à s'interroger sur les motivations profondes de cette munificence dont on sait désormais qu'elle était démesurée. "Sans doute peut-on dire que Timon était aveuglé, ou qu'il s'aveuglait lui-même sur sa capacité à dépenser sans compter. Le prétexte en est l'amitié qu'il porte à tous les Athéniens. Ici, l'amitié est une passion, une raison de vivre, ce qui rend possible la cité véritable» (p. 277). [...] « Timon semble offrir ses dons pour se prouver à lui-même que cette société régie par l'amitié existe réellement, et n'est pas seulement le produit de son ardent désir de la faire exister. Aussi longtemps qu'il est riche, ou qu'il se croit riche, il exclut toute réciprocité. Il ne perçoit pas la fausseté de ses prétendus amis, et s'il faut voir en lui un exemple de démesure, ce ne serait pas à cause d'une générosité sans bornes ni prudence, mais de l'incroyable aveuglement qui lui fait prendre des profiteurs cyniques pour des amis, et une société dont Alcibiade affirme qu'elle est gouvernée par des usuriers pour une société qui mériterait vraiment qu'on se ruine pour elle.

La ruine de Timon découle inexorablement du milieu qui forme son entourage. Un sénateur qui, au début de l'acte II, fait le compte de quelques dettes de Timon peut dire :

Ce gaspillage enragé! Ça ne peut pas durer, ça ne durera pas.

Si je veux de l'or, je n'ai qu'à voler le chien d'un mendiant 
Et le donner à Timon: ce chien devient une mine d'or ;

Si je veux vendre mon cheval et en acheter vingt autres De meilleure race, je donne mon cheval à Timon;

Sans rien demander, je le lui donne, il me pouline aussitôt Plusieurs bêtes, et vigoureuses. Pas de cerbère à sa porte, Mais plutôt un gaillard souriant qui invite sans cesse

Tous les passants. Ça ne peut pas durer ; raisonnablement

Sa situation n'est pas saine.

Le calvaire de Timon va commencer : il ne peut compter sur les sénateurs; il pense qu'il peut compter sur ses amis. Ventidius, qu'il a libéré de prison et dont il a payé la dette en refusant que Ventidius la lui rembourse, refuse de l'aider, alors qu'il a reconnu l'immensité de ce qu'il doit à Timon. Une suite de petites scènes satiriques, cruelles, mordantes, montrent le refus que lui opposent ses amis. Lucullus ? L'heure n'est pas aux prêts, «surtout sur la foi d'une amitié nue, sans garantie » (III.i.42-43). Lucius, lui, s'attend à recevoir quelque cadeau quand arrive le serviteur de Timon. Mais il n'a pas d'argent disponible quand le message de Timon est délivré. [...] Quant à Sempronius, apprenant les refus des autres amis, il feint de s'offusquer que Timon ne se soit pas adressé à lui en premier : «Qui rabaisse mon honneur ne verra pas mon argent» (III.iii.28).

Abandonné de tous — sauf de son Intendant — assailli par les créanciers, Timon, avec rage, s'offre lui-même en pâture : «Décousezmoi jusqu’à la ceinture », «Découpez-moi le cœur», «Comptez mon sang !» ... Et à celui qui réclame cinq mille couronnes : «Cinq mille gouttes paieront cela» (III.iv.89, 91, 93, 95). C'est un écho des allusions au cannibalisme qui ponctuent le premier acte.

Devenu misanthrope, Timon se venge de ses amis en les invitant à un dîner où il leur sert de l'eau tiède, avant de les chasser à coups de pierres. [...] Timon se perd en imprécations dont la violence et la démesure sont souvent comparées à celles du roi Lear. L'ingratitude lui est insupportable. Elle est considérée pendant la Renaissance — qui suivait ici une tradition antique - comme une source potentielle d'impiété envers les dieux, de négligence envers les parents, de trahison envers l'État et d'indifférence envers les amis. C'était une monstrueuse transgression de la loi naturelle, un méprisable exemple de raison dédaignée, et, enfin, une violation de la justice naturelle.

Dans la seconde partie de la pièce, qui se déroule dans une grotte ou autour d'une grotte, Timon reçoit une série de visiteurs et chaque visite est l'occasion d'une surenchère de sa misanthropie. [...] 
Tué par l'ingratitude, révolté par l'injustice, [Timon] ne supporte plus de vivre parmi les hommes, encore qu'il soutienne, contre Apémantus, la supériorité du monde humain sur celui de l'animalité, même s'il souhaite que les bêtes s'emparent de l'empire des hommes.

Les deux misanthropes ne font pas preuve d'une égale démesure : il y a entre eux la différence qu'on faisait naguère entre un révolté et un révolutionnaire : le premier vit aux dépens de la société contre laquelle il se révolte et ne cesse d'avoir besoin d'elle. Le second veut la détruire pour faire place à une société nouvelle : c'est un espoir qu'il n'a pas été donné à Timon d'entretenir. Le caractère radical de son éloignement des hommes ne cessera d'intriguer et d'interpeller les spectateurs ou les lecteurs de la pièce.

On ne peut oublier, cependant, que sa démesure est, si l'on peut dire, à la mesure de sa souffrance et de sa désillusion. Sir Thomas Elyot, l'auteur du traité A Book called The Governor, décrit l'ingratitude comme un vice qui agit d'abord contre la justice : « moste against justice» (The Castell of Health, sig. R8r). Sans justice, sans promesses tenues, sans confiance réciproque, une cité ne peut vraiment survivre que dans le chaos ou la dictature militaire, celle d'Alcibiade, par exemple. Car la pièce s'achève sur une proclamation équivoque du général, qui décide de gouverner en restreignant la paix par la guerre et la guerre par la paix, en se faisant «each other's leech », guérisseur l'un de l'autre (v.iii.85). C'est faire référence à une idée courante à l'époque élisabéthaine, celle de la guerre agissant comme un médecin [leech] contre les humeurs malignes de la paix, et la paix agissant comme médecin contre les blessures de la guerre. Leech signifie aussi sangsue : on peut ainsi concevoir l'ordre comme un équilibre entre deux états maladifs [...], équilibre mécanique ou purement physique obtenu par deux sangsues s'étreignant, ou deux médecins s'entre-guérissant : l'ordre est alors un effet de l'art ou de la violence, et non un produit de la nature, ou de la nature des choses. [...] Comme l'écrivait Hannah Arendt [dans Condition de l'homme moderne], le seul remède à l'imprévisibilité, «à la cahotique incertitude de l'avenir, est contenu dans le pouvoir de faire et de tenir des promesses». Pour Timon, le comportement de ses amis à la fausseté soudain révélée équivaut à un chaos de promesses violées. Car le don accepté, dont le donateur ne doit pas souhaiter la restitution, engage le donataire à une gratitude active. C'est là l'inéluctable 
paradoxe du don. Si l'on perçoit et si l'on estime avec justesse la profondeur du désespoir de Timon, on ne jugera peut-être pas que sa misanthropie est démesurée. [...]

[On peut dire que] Timon d'Athènes [est] une "pure tragédie » au sens où l'entend George Steiner ${ }^{16}$. Non parce que l'humanité y est vile, le cosmos corrompu, les femmes souillées. Mais parce que vers la fin de la pièce, indique Steiner, Timon demande que tout langage prenne fin: «Lips, let four words go by and language end (v.i.219). Ce trope est renforcé du fait que son épitaphe, [ «there does not live a man »], vouée à être effacée par la mer, sera comme la fin de toute textualité. Une telle injonction, poursuit Steiner, ne se trouve nulle part ailleurs dans le corpus: c'est, dans Shakespeare et pour lui, le point zéro - le trou noir — de l'espoir. Une seule fois, une seule, les mots implorent, dans cette œuvre, leur propre anéantissement. » (p. 280283).

\section{Lecture de Shakespeare au XXI $I^{e}$ siècle ${ }^{17}$}

«Les abominations que la tragédie grecque s'était donnée pour tâche de présenter à la cité, quels théâtres, quels spectacles nous en imposent aujourd'hui la vision?

Dans les œuvres de la dernière période de Shakespeare Péricles, Cymbeline, Le conte d'hiver, La tempête - on voit que la réconciliation avec la vie passe par une continuité familiale retrouvée. Et cela suppose, contrairement à l'expérience du malheur au $\mathrm{XX}^{\mathrm{e}}$ siècle, que la collectivité humaine à laquelle appartiennent les protagonistes n'a pas été détruite et abolie. Cela est également vrai de toutes ses tragédies. Parmi-celles-ci, Coriolan et Antoine et Cléopâtre impliquent la sauvegarde de la cité pour la première, et le glorieux règne d'Octave pour la seconde.

Il faut donc s'interroger, quoi qu'il en coûte : la tragédie, telle que Shakespeare la comprenait, n'est-elle pas un genre qui n'a pas encore, aujourd'hui, retrouvé une véritable place parmi nous, sinon comme telle ou telle œuvre du passé que certains archéologues du théâtre nous restituent sans vraiment la faire revivre?

\footnotetext{
${ }^{16}$ George Steiner, «Tragedy, pure and simple », in M.S. Silk, ed., Tragedy and the Tragic Greek Theatre and Beyond, Oxford, 1966, p. 540-541).

17 Texte lu par Dominique Goy-Blanquet.
} 
Il reste qu'à de peu nombreuses, mais' mémorables, occasions la modernité de Shakespeare nous étreint avec une force irrésistible. Indépendamment des productions de metteurs en scène ou de réalisateurs dotés de génie ou d'un talent immense, comme Orson Welles, (Jules César, Othello, Chimes at Midnight [Falstaff]) ; Peter Brooke (Titus Andronicus, Le roi Lear, Timon d'Athènes, Mesure pour mesure); Jean Vilar (Macbeth, Richard II) ; Joseph Mankiewicz (Jules César); les réalisations les plus marquantes ou les plus mémorables sont le fait d'hommes de cinéma ou de théâtre qui ont eu une expérience personnelle de la guerre, du malheur, de la dictature ou du désastre : Erwin Piscator (Le marchand de Venise) ; Giorgio Strehler (Le roi Lear, La tempête) ; Gregori Kosintsev (Hamlet, Le roi Lear), Akira Kurosawa (Le château de l'araignée [d'après Macbeth], Ran [d'après le roi Lear]). La force du texte shakespearien, quand une conscience artistique informée par une expérience aussi vaste que possible est mise à son service, ne laisse pas de resurgir en se renouvelant ${ }^{18}$. »

\footnotetext{
${ }^{18}$ Shakespeare au XXIe siècle, pp. 90-92.
} 